\title{
Scattering kernel for polyatomic molecules
}

\author{
J. Gilbert Méolans* and S. Kokou Dadzie ${ }^{\dagger}$ \\ Université de Provence, UMR CNRS 6595 \\ Département de Mécanique Energétique - IUSTI \\ 5, rue Enrico Fermi 13453 Marseille cedex 13 France
}

(Dated: 08/07/2004)

\begin{abstract}
A polyatomic scattering kernel phenomenologically presented in a previous article is derived from an integral operator formulation. The five parameters involved in the scattering kernel expression are shown to be equal to the accommodation coefficients of various fluxes at the wall, namely: the fluxes of the three components of the momentum and the fluxes of the rotational and vibrational energies of molecules. Under its present form the model is especially convenient for the diatomic molecules.
\end{abstract}

Keywords: rarefied fluid dynamics; molecule-surface impact; Boltzmann equation; kinetic theory; integral equations

\section{INTRODUCTION}

During the last twenty years a need of new knowledge appeared concerning the interaction of gases with solid surfaces in order to formulate realistic boundary conditions in rarefied gas dynamics [1-4]. In spatial research the challenge was especially to predict correct heat fluxes and drag forces on engines reentering in planetary atmospheres. With the recent developments of the gaseous microflows, where the flow fields are characterized by moderately high Knudsen numbers, this topic acquired still more interest. This article is devoted to the derivation of realistic laws linking the distribution functions of the reflected and the

\footnotetext{
*Electronic address: Gilbert. Meolans@polytech.univ-mrs.fr

${ }^{\dagger}$ Electronic address: Kokou.Dadzie@polytech.univ-mrs.fr; URL: http://www.polytech.univ-mrs.fr
} 
incoming particles at the wall. As it is well known such laws may be used as boundary conditions in order to resolve the Boltzmann equation. Moreover, in the slip regime, these laws also allow to obtain more accurate velocity slip and temperature jumps at the wall: so the validity domain of the continuum equations is extended up to higher Knudsen numbers when these equations are associated to the correct boundary conditions.

In a previous article [5] we developed a model of a scattering kernel for unstructured molecules using an integral operator formulation as illustrated by Cercignani [6, 7]. At the end of this previous work the proposed scattering kernel was extended to the case of molecules with internal structure on the basis of brief phenomenological arguments. In the present article, the integral operator formulation is generalized to a polyatomic gas: then the polyatomic scattering kernel is methodologically derived from the study of an eigenvalue equation and the meaning of the five parameters introduced in the kernel is clarified.

In section II we deduced the polyatomic scattering kernel, investigating the associated integral operator. In its fully developed form the scattering kernel appears as a linear combination of 32 partial kernels declining all the possible associations of, respectively, diffusive and specular processes (according to three directions) and elastic or inelastic processes (for the internal modes). The 32 coefficients of this combination are the weight of the various accommodation processes and they depend on five basic parameters. These five parameters are shown to be respectively equal to the accommodation coefficients of the momentum components and of the internal energies. In section III, we present a general comment on this method of integral operator in the framework of scattering kernel derivation.

\section{POLYATOMIC SCATTERING KERNEL DERIVATION.}

We consider the problem of finding the scattering kernel,

$$
B\left(V^{\prime}, E_{i r^{\prime}}, E_{i v^{\prime}}, g_{i r^{\prime}}, V, E_{i r}, E_{i v}, g_{i r}\right)
$$

governing the reflection of polyatomic molecules at the wall. $V^{\prime}$ is the velocity of the impinging gas particle referred to the wall, $V^{\prime}=\left(V_{x}^{\prime}, V_{y}^{\prime}, V_{z}^{\prime}\right) \in\left\{\Omega^{\prime}=\Omega_{x}^{\prime} \times \Omega_{y}^{\prime} \times \Omega_{z}^{\prime}=\mathbb{R}_{-} \times \mathbb{R} \times \mathbb{R}\right\}$ 
and $V$ the velocity of the reflected one referred to the wall, $V=\left(V_{x}, V_{y}, V_{z}\right) \in\{\Omega=$ $\left.\Omega_{x} \times \Omega_{y} \times \Omega_{z}=\mathbb{R}_{+} \times \mathbb{R} \times \mathbb{R}\right\}$. These velocities reduce to the peculiar velocities when the slip velocity at the wall is neglected. $V_{R}$ is defined as $V_{R}=\left(-V_{x}, V_{y}, V_{z}\right)$, and $(x, y, z)$ are the three spatial coordinates with $x$ the normal axis to the wall oriented from the wall towards the gas. $E_{i r^{\prime}}$ and $E_{i v^{\prime}}$ are respectively the rotational energy and the vibrational energy of an incident particle at the wall. Similarly $E_{i r}$ and $E_{i v}$ are respectively the rotational energy and the vibrational energy of a reflected particle at the wall. Then the subscripts ${ }_{i r}$ and ${ }_{i v}$ are the quantum numbers related to the internal energy of a particle, hence they are integers; $g_{i r}$ is the weight of the rotational degeneracy and will be taken here equal to $(2 i r+1)$. The kernel $B$, which is the density of probability that a molecule in a state $\left(V^{\prime}, E_{i r^{\prime}}, E_{i v^{\prime}}\right)$ hitting the wall at any point $X$ of the wall is reflected at the same point in a state $\left(V, E_{i r}, E_{i v},\right)$, must satisfy the following conditions detailed in reference [5]. [1-3]:

the non-negativity,

$$
B\left(V^{\prime}, E_{i r^{\prime}}, E_{i v^{\prime}}, g_{i r^{\prime}}, V, E_{i r}, E_{i v}, g_{i r}\right) \geq 0
$$

the normalization,

$$
\sum_{i r, i v} \int_{\Omega} B\left(V^{\prime}, E_{i r^{\prime}}, E_{i v^{\prime}}, g_{i r^{\prime}}, V, E_{i r}, E_{i v}, g_{i r}\right) d_{V}=1
$$

and the reciprocity relation,

$$
\begin{gathered}
\left|V_{x}^{\prime}\right| e^{-\frac{\left\|V^{\prime}\right\|^{2}}{C_{w}^{2}}} e^{-\varepsilon_{i r^{\prime}}} e^{-\varepsilon_{i v^{\prime}}} g_{i r^{\prime}} B\left(V^{\prime}, E_{i r^{\prime}}, E_{i v^{\prime}}, V, E_{i r}, E_{i v}\right)= \\
V_{x} e^{-\frac{\|V\|^{2}}{C_{w}^{2}}} e^{-\varepsilon_{i r}} e^{-\varepsilon_{i v}} g_{i r} B\left(-V, E_{i r}, E_{i v},-V^{\prime}, E_{i r^{\prime}}, E_{i v^{\prime}}\right)
\end{gathered}
$$

where

$$
\varepsilon_{i r}=\frac{E_{i r}}{k T_{w}}, \quad \varepsilon_{i v}=\frac{E_{i v}}{k T_{w}}
$$

with $k$ the Boltzmann constant and $T_{w}$ the wall temperature. 


\section{A. Analytical derivation from integral operator}

Let us write the transformation

$$
\begin{array}{r}
K\left(V, E_{i r}, E_{i v}, g_{i r}, V^{\prime}, E_{i r^{\prime}}, E_{i v^{\prime}}, g_{i r^{\prime}}\right)=\left[\left|V_{x}^{\prime}\right| f_{0}\left(V^{\prime}, E_{i r^{\prime}}, E_{i v^{\prime}}\right)\right]^{\frac{1}{2}}\left[\left|V_{x}\right| f_{0}\left(V, E_{i r}, E_{i v}\right)\right]^{\frac{-1}{2}} \\
B\left(V_{R}^{\prime}, E_{i r^{\prime}}, E_{i v^{\prime}}, g_{i r^{\prime}}, V, E_{i r}, E_{i v}, g_{i r}\right)
\end{array}
$$

where $f_{0}\left(V, E_{i r}, E_{i v}\right)$ is the local equilibrium distribution function at the temperature $T_{w}$ of the wall, defined by:

$$
f_{0}\left(V, E_{i r}, E_{i v}\right)=\frac{n}{\left(C_{w} \sqrt{\pi}\right)^{3}} e^{-\frac{\|V\|^{2}}{C_{w}^{2}}} \frac{g_{i r} e^{-\varepsilon_{i r}} e^{-\varepsilon_{i v}}}{Q_{r} Q_{v}}
$$

with

$$
Q_{r}=\sum_{i r} g_{i r} e^{-\varepsilon_{i r}}, \quad Q_{v}=\sum_{i v} e^{-\varepsilon_{i v}}, \quad C_{w}^{2}=\frac{2 k T_{w}}{m} .
$$

Since $f_{0}$ is a known function the problem of finding $B$ is equivalent to finding $K$. The normalization condition and the non-negativity conditions on $B$ imply obviously the same conditions on $K$.

Note $\digamma_{r}$ the set of the rotational energy states $E_{i r}$, and $\digamma_{v}$ the set of the vibrational energy states $E_{i v}$. Consider the five elementary Hilbert spaces of states $L^{2}\left(\Omega_{\kappa}\right)_{\kappa=x, y, z}, L^{2}\left(\digamma_{r}\right)$, and $L^{2}\left(\digamma_{v}\right)$ of square summable functions with their corresponding usual scalar product

$$
\begin{aligned}
& <\varphi_{\kappa 1}, \varphi_{\kappa 2}>_{\kappa}=\int_{\Omega_{\kappa}} \varphi_{\kappa 1}(V) \varphi_{\kappa 2}(V) d_{V}, \text { for all } \varphi_{\kappa 1}, \varphi_{\kappa 2} \in L^{2}\left(\Omega_{\kappa}\right), \kappa=x, y, z \\
& <\varphi_{r 1}, \varphi_{r 2}>_{r}=\sum_{i r} \varphi_{r 1}\left(E_{i r}\right) \varphi_{r 2}\left(E_{i r}\right), \text { for all } \varphi_{r 1}, \varphi_{r 2} \in L^{2}\left(\digamma_{r}\right) \\
& <\varphi_{v 1}, \varphi_{v 2}>_{v}=\sum_{i v} \varphi_{v 1}\left(E_{i v}\right) \varphi_{v 2}\left(E_{i v}\right), \text { for all } \varphi_{v 1}, \varphi_{v 2} \in L^{2}\left(\digamma_{v}\right)
\end{aligned}
$$

Consider The tensor product $£=L^{2}\left(\Omega_{x}\right) \otimes L^{2}\left(\Omega_{y}\right) \otimes L^{2}\left(\Omega_{z}\right) \otimes L^{2}\left(\digamma_{r}\right) \otimes L^{2}\left(\digamma_{v}\right)$ of the five Hilbert spaces of states. Let us remark that this tensor product of Hilbert space $£$ is dense in the Hilbert space $\mathcal{H}=L^{2}(\Omega) \otimes L^{2}\left(\digamma_{r}\right) \otimes L^{2}\left(\digamma_{v}\right)$ where the scalar product is defined by

$$
<\varphi_{1}, \varphi_{2}>=\sum_{i r, i v} \int_{\Omega} \varphi_{1}\left(V, E_{i r}, E_{i v}\right) \varphi_{2}\left(V, E_{i r}, E_{i v}\right) d_{V} \text { for all } \varphi_{1}, \varphi_{2} \in \mathcal{H}
$$

Instead of studying the problem of the kernel $K$, we study the linear integral associated operator $A$ defined on $\mathcal{H}$ by

$$
A(\psi)=\sum_{i r^{\prime}, i v^{\prime}} \int_{\Omega^{\prime}} K\left(V, E_{i r}, E_{i v}, g_{i r}, V^{\prime}, E_{i r^{\prime}}, E_{i v^{\prime}}, g_{i r^{\prime}}\right) \psi\left(V^{\prime}, E_{i r^{\prime}}, E_{i v^{\prime}}, g_{i r^{\prime}}\right) d_{V^{\prime}}
$$


Assume that the operator $A$ has a purely discrete spectrum, and assume that its eigenfunctions are all in the Hilbert space $£$. The kernel $K$ can be written in the form:

$$
\begin{array}{r}
\sum_{j_{x}, j_{y}, j_{z}, j_{r}, j_{v}=0}^{\infty} \lambda_{j_{x}, j_{y}, j_{z}, j_{r}, j_{v}} \psi_{j_{x}}\left(V_{x}\right) \psi_{j_{y}}\left(V_{y}\right) \psi_{j_{z}}\left(V_{z}\right) \psi_{j_{r}}\left(E_{i r}\right) \psi_{j_{v}}\left(E_{i v}\right) \\
\psi_{j_{x}}\left(V_{x}^{\prime}\right) \psi_{j_{y}}\left(V_{y}^{\prime}\right) \psi_{j_{z}}\left(V_{z}^{\prime}\right) \psi_{j_{r}}\left(E_{i r^{\prime}}\right) \psi_{j_{v}}\left(E_{i v^{\prime}}\right)
\end{array}
$$

where the functions $\psi_{j_{x}}\left(V_{x}\right) \psi_{j_{y}}\left(V_{y}\right) \psi_{j_{z}}\left(V_{z}\right) \psi_{j_{r}}\left(E_{i r}\right) \psi_{j_{v}}\left(E_{i v}\right)$ are the eigenfunctions of $A$ with their corresponding eigenvalues $\lambda_{j_{x}, j_{y}, j_{z}, j_{r}, j_{v}}$. According to the non-negativity and the normalization conditions, the eigenvalues must satisfy $\lambda_{j_{x}, j_{y}, j_{z}, j_{r}, j_{v}} \in[0,1]$ for all $j_{x}, j_{y}, j_{z}, j_{r}, j_{v} \in$ $\mathbb{N}$. Moreover, one can see that, in the tensor product space $£$, the scalar product (8) equals the scalar product defined in this tensor product space $£$ by the product of the five elementary scalar products (8). So, we can suppose that the eigenvalues have the form $\lambda_{j_{x}} \lambda_{j_{y}} \lambda_{j_{z}} \lambda_{j_{r}} \lambda_{j_{v}}$ and that the set of functions $\psi_{j_{\chi}}\left(M_{\chi}\right), j_{\chi} \in \mathbb{N}$, is a function basis of the $\chi$ corresponding Hilbert space, $\chi=x, y, z, r, v$. Therefore, the expression (10) can be written as a product of five infinite sums:

$$
K=\prod_{\chi \in\{x, y, z, r, v\}} \sum_{j=0}^{\infty} \lambda_{j_{\chi}} \psi_{j_{\chi}}\left(M_{\chi}\right) \psi_{j_{\chi}}\left(M_{\chi}^{\prime}\right), \quad M_{\chi}=V_{x}, V_{y}, V_{z}, E_{i r}, E_{i v}
$$

Define $\psi_{0}=\psi_{0_{x}} \psi_{0_{y}} \psi_{0_{z}} \psi_{0_{r}} \psi_{0_{v}}$ by

$$
\begin{array}{r}
\psi_{0_{x}}\left(V_{x}\right)=\frac{\sqrt{2}}{C_{w}}\left|V_{x}\right|^{\frac{1}{2}} e^{\frac{-V_{x}^{2}}{2 C_{w}^{2}}}, \quad \psi_{0_{y}}\left(V_{y}\right)=\left(C_{w} \sqrt{\pi}\right)^{\frac{-1}{2}} e^{\frac{-V_{y}^{2}}{2 C_{w}^{2}}}, \\
\psi_{0_{z}}\left(V_{z}\right)=\left(C_{w} \sqrt{\pi}\right)^{\frac{-1}{2}} e^{\frac{-V_{z}^{2}}{2 C_{w}^{2}}}, \quad \psi_{0_{r}}\left(E_{i r}\right)=\sqrt{\frac{g_{i r}}{Q_{r}}} e^{-\frac{1}{2} \epsilon_{i r}}, \quad \psi_{0_{v}}\left(E_{i v}\right)=\frac{e^{-\frac{1}{2} \epsilon_{i v}}}{\sqrt{Q_{v}}} .
\end{array}
$$

Let us prove that $\psi_{0}$, is an eigenfunction of $A$. Mathematically, the normalization condition can be also written

$$
\sum_{i r^{\prime}, i v^{\prime}} \int_{\Omega^{\prime}} B\left(-V, E_{i r}, E_{i v}, g_{i r},-V_{R}^{\prime}, E_{i r^{\prime}}, E_{i v^{\prime}}, g_{i r^{\prime}}\right) d_{V^{\prime}}=1
$$

from this relation (12), the reciprocity relation (3) leads to:

$$
\sum_{i r^{\prime}, i v^{\prime}} \int_{\Omega^{\prime}}\left|V_{x}^{\prime}\right| f_{0}\left(V^{\prime}, E_{i r^{\prime}}, E_{i v^{\prime}}\right) B\left(V_{R}^{\prime}, E_{i r^{\prime}}, E_{i v^{\prime}}, g_{i r^{\prime}}, V, E_{i r}, E_{i v}, g_{i r}\right) d_{V^{\prime}}=\left|V_{x}\right| f_{0}\left(-V, E_{i r}, E_{i v}\right)(13)
$$


Using the relation (13), the calculation of $A\left(\psi_{0}\right)$ gives $A\left(\psi_{0}\right)=\psi_{0}$. Consequently $\psi_{0}=$ $\psi_{0_{x}} \psi_{0_{y}} \psi_{0_{z}} \psi_{0_{r}} \psi_{0_{v}}$ is an eigenfunction of the operator $A$ associated to the eigenvalue 1 .

Now, following the five state parameters, let us introduce five parameters related to the eigenvalues as follows: $\lambda_{0_{\chi}}=1$, and for $j \neq 0, \lambda_{j_{\chi}}=\left(1-\alpha_{\chi}\right)$ for all $\chi=x, y, z, r, v$. The relation (11) becomes

$$
K=\prod_{\chi \in\{x, y, z, r, v\}}\left[\psi_{0_{\chi}}\left(M_{\chi}\right) \psi_{0_{\chi}}\left(M_{\chi}^{\prime}\right)+\left(1-\alpha_{\chi}\right) \sum_{j=1}^{\infty} \psi_{j_{\chi}}\left(M_{\chi}\right) \psi_{j_{\chi}}\left(M_{\chi}^{\prime}\right)\right]
$$

which may be written

$$
K=\prod_{\chi \in\{x, y, z, r, v\}}\left[\alpha_{\chi} \psi_{0_{\chi}}\left(M_{\chi}\right) \psi_{0_{\chi}}\left(M_{\chi}^{\prime}\right)+\left(1-\alpha_{\chi}\right) \sum_{j=0}^{\infty} \psi_{j_{\chi}}\left(M_{\chi}\right) \psi_{j_{\chi}}\left(M_{\chi}^{\prime}\right)\right]
$$

Finally, using the following property

$$
\sum_{j=0}^{\infty} \psi_{j_{\chi}}\left(M_{\chi}\right) \psi_{j_{\chi}}\left(M_{\chi}^{\prime}\right)=\delta\left(M_{\chi}-M_{\chi}^{\prime}\right)
$$

where $\delta$ is the dirac function. It is obtained

$$
\begin{array}{r}
K=\left\{\alpha_{x} \psi_{0_{x}}\left(V_{x}\right) \psi_{0_{x}}\left(V_{x}^{\prime}\right)+\left(1-\alpha_{x}\right) \delta\left(V_{x}-V_{x}^{\prime}\right)\right\}\left\{\alpha_{y} \psi_{0_{y}}\left(V_{y}\right) \psi_{0_{y}}\left(V_{y}^{\prime}\right)+\left(1-\alpha_{y}\right) \delta\left(V_{y}-V_{y}^{\prime}\right)(14)\right. \\
\left\{\alpha_{z} \psi_{0_{z}}\left(V_{z}\right) \psi_{0_{z}}\left(V_{z}^{\prime}\right)+\left(1-\alpha_{z}\right) \delta\left(V_{z}-V_{z}^{\prime}\right)\right\}\left\{\alpha_{r} \psi_{0_{r}}\left(V_{r}\right) \psi_{0_{r}}\left(V_{r}^{\prime}\right)+\left(1-\alpha_{r}\right) \delta\left(V_{r}-V_{r}^{\prime}\right)\right\} \\
\left\{\alpha_{v} \psi_{0_{v}}\left(V_{v}\right) \psi_{0_{v}}\left(V_{v}^{\prime}\right)+\left(1-\alpha_{v}\right) \delta\left(V_{v}-V_{v}^{\prime}\right)\right\} .
\end{array}
$$

Applying inversely the transformation (5), the operator $B$ corresponding to the kernel $K$ above (relationship (14)) is

$$
\begin{gathered}
B\left(V^{\prime}, E_{i r^{\prime}}, E_{i v^{\prime}}, g_{i r^{\prime}}, V, E_{i r}, E_{i v}, g_{i r}\right)=\left\{\left(1-\alpha_{x}\right) \delta\left(V_{x}^{\prime}+V_{x}\right)+\alpha_{x} \frac{2 V_{x}}{C_{w}^{2}} e^{-\frac{V_{x}^{2}}{C_{w}^{2}}}\right\} \\
\left\{\left(1-\alpha_{y}\right) \delta\left(V_{y}^{\prime}-V_{y}\right)+\alpha_{y} \frac{1}{C_{w} \sqrt{\pi}} e^{-\frac{V_{y}^{2}}{C_{w}^{2}}}\right\}\left\{\left(1-\alpha_{y}\right) \delta\left(V_{z}^{\prime}-V_{z}\right)+\alpha_{z} \frac{1}{C_{w} \sqrt{\pi}} e^{-\frac{V_{z}^{2}}{C_{w}^{2}}}\right\} \\
\left\{\left(1-\alpha_{r}\right) \delta\left(E_{i r^{\prime}}-E_{i r}\right)+\alpha_{r} \frac{g_{i r}}{Q_{r}} e^{-\epsilon_{i r}}\right\}\left\{\left(1-\alpha_{v}\right) \delta\left(E_{i v^{\prime}}-E_{i v}\right)+\alpha_{v} \frac{1}{Q_{v}} e^{-\epsilon_{i v}}\right\} .
\end{gathered}
$$

In the further calculations, we will note the scattering kernel (15) simply

$$
B=P_{x} P_{y} P_{z} P_{r} P_{v}
$$


where $P_{x}, P_{y}, P_{z}, P_{r}, P_{v}$ correspond respectively to the five factors of the expression (15). It would be seen that these five factors satisfy

$$
\int_{0}^{+\infty} P_{x} d_{V_{x}}=\int_{-\infty}^{+\infty} P_{y} d_{V_{y}}=\int_{-\infty}^{+\infty} P_{z} d_{V_{z}}=\sum_{i r} P_{r}=\sum_{i v} P_{v}=1 .
$$

On the other hand when developing the expression (15) one obtains the kernel $B$ as combination of 32 elementary scattering kernels where the coefficients are functions of the $\alpha_{\chi}$.

\section{B. On the coefficient $\alpha_{\chi}$}

In this section we prove that the five coefficients $\alpha_{\chi}$ involved in the scattering kernel equal respectively the accommodation coefficients of the various fluxes of the five microscopic state parameters $\left(M_{\chi}=V_{x}, V_{y}, V_{z}, E_{i r}, E_{i v}\right)$.

The accommodation coefficient $\beta_{\chi}$ of a physical property $M_{\chi}$ at the wall is defined through the relation $[3,4,8]$ :

$$
\beta_{\chi}=\frac{\Phi_{\chi}^{-}-\Phi_{\chi}^{+}}{\Phi_{\chi}^{-}-\Phi_{\chi}^{e}}
$$

where $\Phi_{\chi}^{-}$is the incoming flux at the wall of the property $M_{\chi}, \Phi_{\chi}^{+}$is the corresponding reflected flux, and $\Phi_{\chi}^{e}$ is the reflected flux in the hypothetical situation of perfect accommodation to the wall. These various fluxes are written:

$$
\begin{gathered}
\Phi_{\chi}^{-}=\sum_{i r^{\prime}, i v^{\prime}} \int_{\Omega^{\prime}} m\left|V_{x}^{\prime}\right| M_{\chi}^{\prime} f^{-}\left(V^{\prime}, E_{i r^{\prime}}, E_{i v^{\prime}}, g_{i r^{\prime}}\right) d_{V^{\prime}} \\
\Phi_{\chi}^{+}=\sum_{i r, i v} \int_{\Omega} m\left|V_{x}\right| M_{\chi} f^{+}\left(V, E_{i r}, E_{i v}, g_{i r}\right) d_{V}
\end{gathered}
$$

where $f^{-}$and $f^{+}$are respectively the incident and the reflected distribution functions linked by the relation

$$
\begin{array}{r}
\left|V_{x}\right| f_{i}^{+}\left(V, E_{i r}, g_{i r}, E_{i v}\right)= \\
\sum_{i r^{\prime}, i v^{\prime}} \int_{\Omega^{\prime}}\left|V_{x}^{\prime}\right| f^{-}\left(V^{\prime}, E_{i r^{\prime}}, g_{i r^{\prime}}, E_{i v^{\prime}}\right) B\left(V^{\prime}, E_{i r^{\prime}}, g_{i r^{\prime}}, E_{i v^{\prime}}, V, E_{i r}, g_{i r}, E_{i v}\right) d_{V^{\prime}}
\end{array}
$$


Accounting for (20), the reflected flux $\Phi_{\chi}^{+}$(expression (19))may be rewritten:

$$
\Phi_{\chi}^{+}=\sum_{i r^{\prime}, i v^{\prime}} \int_{\Omega^{\prime}} m\left|V_{x}^{\prime}\right| f^{-}\left(V^{\prime}, E_{i r^{\prime}}, g_{i r^{\prime}}, E_{i v^{\prime}}\right)\left[\sum_{i r, i v} \int_{\Omega} M_{\chi} P_{x} P_{y} P_{z} P_{r} P_{v} d_{V}\right] d_{V^{\prime}}
$$

and the reflected flux in the case of perfect accommodation is written

$$
\Phi_{\chi}^{e}=\sum_{i r^{\prime}, i v^{\prime}} \int_{\Omega^{\prime}} m\left|V_{x}^{\prime}\right| f^{-}\left(V^{\prime}, E_{i r^{\prime}}, g_{i r^{\prime}}, E_{i v^{\prime}}\right)\left[\sum_{i r, i v} \int_{\Omega} M_{\chi} B_{e} d_{V}\right] d_{V^{\prime}}
$$

where $B_{e}$ the perfect accommodation scattering kernel is defined by

$$
B_{e}=\frac{2 g_{i r}}{Q_{r} Q_{v} C_{w}^{4} \pi} V_{x} e^{-\frac{\|V\|^{2}}{C_{w}^{2}}} e^{-\epsilon_{i r}} e^{-\epsilon_{i v}}
$$

\section{1. calculation of $\beta_{y}$ and $\beta_{z}$}

The tangential accommodation coefficient, $\beta_{y}$ is obtained by substituting $M_{\chi}=V_{y}$ in the definition relation (17). In this case it is easily seen that $\Phi_{y}^{e}=0$. Then accounting for the property (16) the expression (21) leads to

$$
\Phi_{y}^{+}=\left(1-\alpha_{y}\right) \sum_{i r^{\prime}, i v^{\prime}} \int_{\Omega^{\prime}} m V_{y}^{\prime}\left|V_{x}^{\prime}\right| f^{-}\left(V^{\prime}, E_{i r^{\prime}}, g_{i r^{\prime}}, E_{i v^{\prime}}\right) d_{V^{\prime}} .
$$

It results immediately from expression (17)

$$
\beta_{y}=1-\frac{\Phi_{y}^{+}}{\Phi_{y}^{-}}=\alpha_{y}
$$

Similarly it is found

$$
\beta_{z}=1-\frac{\Phi_{z}^{+}}{\Phi_{z}^{-}}=\alpha_{z}
$$

\section{2. calculation of $\beta_{x}$}

The normal accommodation coefficient is obtained by substituting $M_{\chi}=\left|V_{x}\right|$ in the definition (17). In this case, accounting for the property (16) and the expression of the partial operator $P_{x}$, it is obtained

$$
\sum_{i r, i v} \int_{\Omega}\left|V_{x}\right| P_{x} P_{y} P_{z} P_{r} P_{v} d_{V}=-\left(1-\alpha_{x}\right) V_{x}^{\prime}+\alpha_{x} \frac{C_{w} \sqrt{\pi}}{2}
$$


then the expression (21) yields

$$
\Phi_{x}^{+}-\Phi_{x}^{-}=\alpha_{x} \sum_{i r^{\prime}, i v^{\prime}} \int_{\Omega^{\prime}} m\left|V_{x}^{\prime}\right| f^{-}\left(V^{\prime}, E_{i r^{\prime}}, E_{i v^{\prime}}, g_{i r^{\prime}}\right)\left(V_{x}^{\prime}+\frac{C_{w} \sqrt{\pi}}{2}\right) d_{V^{\prime}} .
$$

The calculation of $\Phi^{e}$ leads easily to

$$
\Phi_{x}^{e}-\Phi_{x}^{-}=\sum_{i r^{\prime}, i v^{\prime}} \int_{\Omega^{\prime}} m\left|V_{x}^{\prime}\right| f^{-}\left(V^{\prime}, E_{i r^{\prime}}, E_{i v^{\prime}}, g_{i r^{\prime}}\right)\left(V_{x}^{\prime}+\frac{C_{w} \sqrt{\pi}}{2}\right) d_{V^{\prime}} .
$$

Consequently we obtained from the relation (17)

$$
\beta_{x}=\alpha_{x}
$$

3. calculation of $\beta_{r}$ and $\beta_{v}$

Now substitute $M_{\chi}=g_{i r} E_{i r}$ in the relation (17). Accounting for the property (16) it is obtained

$$
\sum_{i r, i v} \int_{\Omega} g_{i r} E_{i r} P_{x} P_{y} P_{z} P_{r} P_{v} d_{V}=\sum_{i r} g_{i r} E_{i r} P_{r}=\left(1-\alpha_{r}\right) g_{i r^{\prime}} E_{i r^{\prime}}+\alpha_{r} \frac{Q_{r}^{*}}{Q_{r}}
$$

where we have noted

$$
Q_{r}^{*}=\sum_{i r} g_{i r}^{2} E_{i r} e^{-\varepsilon_{i r}}
$$

Then the expression of $\Phi^{+}$leads to

$$
\Phi_{E_{i r}}^{-}-\Phi_{E_{i r}}^{e}=\alpha_{r} \sum_{i r^{\prime}, i v^{\prime}} \int_{\Omega^{\prime}} m\left|V_{x}^{\prime}\right| f^{-}\left(V^{\prime}, E_{i r^{\prime}}, E_{i v^{\prime}}, g_{i r^{\prime}}\right)\left(g_{i r^{\prime}} E_{i r^{\prime}}-\frac{Q_{r}^{*}}{Q_{r}}\right) d_{V^{\prime}} .
$$

Using the expression (23) of $B_{e}$, we obtain

$$
\sum_{i r, i v} \int_{\Omega} g_{i r} E_{i r} B_{e} d_{V}=\frac{Q_{r}^{*}}{Q_{r}}
$$

and then

$$
\Phi_{E_{i r}}^{-}-\Phi_{E_{i r}}^{e}=\sum_{i r^{\prime}, i v^{\prime}} \int_{\Omega^{\prime}} m\left|V_{x}^{\prime}\right| f^{-}\left(V^{\prime}, E_{i r^{\prime}}, E_{i v^{\prime}}, g_{i r^{\prime}}\right)\left(g_{i r^{\prime}} E_{i r^{\prime}}-\frac{Q_{r}^{*}}{Q_{r}}\right) d_{V^{\prime}}
$$

consequently,

$$
\beta_{r}=\alpha_{r}
$$


In the same way, substituting $M_{\chi}=E_{i v}$, it is found

$$
\beta_{v}=\alpha_{v}
$$

In conclusion, the five parameters $\alpha_{\chi}$ involved in the scattering kernel (15) are the accommodation coefficients corresponding to the five state parameters, namely the three momentum components and the two internal energy degrees.

\section{COMMENT ON THE METHOD USED IN SCATTERING KERNEL DERIVATION}

The $\mathcal{H}$ Hilbert space corresponds generally to the Hilbert space used in the framework of the modelling of the Boltzmann equation in polyatomic gases. Following the quantum mechanic concept, the wall and then the boundary conditions can be represented by an operator defined on this $\mathcal{H}$ Hilbert space $[4,6,8,9]$. Therefore the problem of boundary condition for the Boltzmann equation can be basically formulated through the integral operator (9), so this formulation is convenient for solving the linearized Boltzmann equation. The reciprocity relation assumption globally means that the local equilibrium distribution function must be invariant by the kernel $B[8,9]$. This last condition, which contains thermodynamic properties is the most important condition. In addition, it is the only one condition containing physical meaning. This condition leads to the first eigenfunction representation $\psi_{0}$. The other eigenfunctions remain unknown. At this step of the scattering kernel construction, one should suggest to choose the set of the other eigenfunctions [10] or a finite number of them. But, such a way would be a purely mathematical construction without real physical justifications $[4,7]$.

Then, another way consists to analyze physically the accommodation process. Let us consider the couple gas/surface characterized by its macroscopic properties. It seems convenient to assume that for each microscopic property $p$ of the molecules, the solid surface behaves in a way perfectly defined in the accommodation process. In other worlds, in a gas/surface configuration, physically and geometrically given, for each microscopic property 
there is a linear relation between the amount of the $p$ flux accommodated by the wall and the amount of the $p$ incoming flux. So the accommodation at the wall of any physical microscopic property provides a physical information through a corresponding accommodation coefficient. Thus, five elementary accommodation coefficients associated to the five basic parameters defining the molecule states, (the three momentum components, rotational energy, vibrational energy) are naturally introduced completely describing the molecules behavior in the reflection process. In this way, the present construction of the polyatomic scattering kernel is based on five accommodation coefficients, and corresponds to an integral operator partially degenerated involving 32 different eigenvalues in its expansion: from our point of view, this construction appears physically founded and completely describing the reflection process.

\section{CONCLUDING REMARKS}

We have established a scattering kernel to structured molecules involving one rotational and one vibrational energy mode. A convenient integral operator formulation is used assuming a purely discrete spectrum and assuming eigenvalues depending on five basic parameters in respect to the five state parameters of the molecules (and then assuming a partial degeneracy of the integral operator). These five basic parameters are shown to be the accommodation coefficients of, respectively, the three momentum accommodation coefficients and the accommodation coefficients of the two internal energy modes.

Under its factorized form (15), the proposed scattering kernel is easy to use in analytical calculations or to be implemented in numerical modelling. In order to show its physical meaning, the expression (15) may be developed. Under its developed form, the scattering kernel appears as a linear combination of 32 elementary scattering kernels (listed in the appendix). All these elementary kernels correspond to various situations of accommodation at the wall which have been described in reference [5]. The linear combination coefficients, which represent the weight of the various types of accommodation in the reflection process, 
are combinations of the factors $\alpha_{\chi}$ and $\left(1-\alpha_{\chi}\right)$ (see the Appendix). In each elementary kernel each molecule state accommodates independently from the others. So the new kernel allows to take into account the interplay between the molecule freedom degrees when interacting at the wall [11]. Up to now, the data available concerning the whole set of accommodation coefficients involved in the proposed scattering kernel are rare: that makes a complete validation of the model difficult.

Finally, let us add that, in the form presented here, the scattering kernel accounts for a single rotational and a single vibrational mode. This description is sufficient in any condition for diatoms. In the case of more complex polyatomic structures, involving various vibrational (or rotational) modes, the present form of scattering kernel remains directly usable, as long as the various vibrational (or rotational) modes remain in a same thermodynamics state (i.e in local equilibrium the ones with the others). In a contrary situation (for example in strong vibrational nonequilibrium conditions) it may be pertinent - depending on the considered time scale - to distinguish various vibrational (or rotational) accommodation coefficients to describe the reflection process. In such a case, the scattering kernel should be written in the same way as previously, but it should involve more than five state parameters, and thus more than five accommodation coefficients.

\section{APPENDIX A: DIFFERENT WRITING OF THE SCATTERING KERNEL $B$ OF RELATION (15)}

Let

$$
\begin{aligned}
& \tilde{P}_{0}=\delta\left(E_{i r^{\prime}}-E_{i r}\right) \delta\left(E_{i v^{\prime}}-E_{i v}\right), \quad \tilde{P}_{r v}=\frac{e^{-\varepsilon_{i r}}}{Q_{r}} g_{i r} \frac{e^{-\varepsilon_{i v}}}{Q_{v}} \\
& \tilde{P}_{v}=\delta\left(E_{i r^{\prime}}-E_{i r}\right) \frac{e^{-\varepsilon_{i v}}}{Q_{v}}, \quad \tilde{P}_{r}=\frac{e^{-\varepsilon_{i r}}}{Q_{r}} g_{i r} \delta\left(E_{i v^{\prime}}-E_{i v}\right)
\end{aligned}
$$

Develop partially the expression (15). The scattering kernel can be written in the form presented in reference [5], as follows: 


$$
\begin{array}{r}
B=\left(\sum_{\kappa} \mu_{\kappa} B_{\kappa}\left(V^{\prime}, V\right)\right)\left(\left(1-\alpha_{r}\right)\left(1-\alpha_{v}\right) \tilde{P}_{0}+\right. \\
\left.\alpha_{v}\left(1-\alpha_{r}\right) \tilde{P}_{v}+\alpha_{r}\left(1-\alpha_{v}\right) \tilde{P}_{r}+\alpha_{v} \alpha_{r} \tilde{P}_{r v}\right)
\end{array}
$$

where $B_{\kappa}$ the elementary scattering kernels, and $\mu_{\kappa}$ their corresponding coefficients in the case of unstructured molecule given in reference [5], are recalled below:

$$
\begin{gathered}
B_{0}\left(V^{\prime}, V\right)=\delta\left(V_{x}+V_{x}^{\prime}\right) \delta\left(V_{y}-V_{y}^{\prime}\right) \delta\left(V_{z}-V_{z}^{\prime}\right) \\
B_{y z}\left(V^{\prime}, V\right)=\frac{1}{\pi C_{w}^{2}} \delta\left(V_{x}+V_{x}^{\prime}\right) e^{-\frac{V_{y}^{2}}{C_{w}^{2}}} e^{-\frac{V_{z}^{2}}{C_{w}^{2}}}, \\
B_{x z}\left(V^{\prime}, V\right)=\frac{2}{C_{w}^{3} \sqrt{\pi}} V_{x} \delta\left(V_{y}-V_{y}^{\prime}\right) e^{-\frac{V_{x}^{2}}{C_{w}^{2}}} e^{-\frac{V_{z}^{2}}{C_{w}^{2}}}, \\
B_{x y}\left(V^{\prime}, V\right)=\frac{2}{C_{w}^{3} \sqrt{\pi}} V_{x} \delta\left(V_{z}-V_{z}^{\prime}\right) e^{-\frac{V_{x}^{2}}{C_{w}^{2}}} e^{-\frac{V_{y}^{2}}{C_{w}^{2}}}, \\
B_{x y z}\left(V^{\prime}, V\right)=\frac{2}{\pi C_{w}^{4}} V_{x} e^{-\frac{V_{x}^{2}}{C_{w}^{2}}} e^{-\frac{V_{y}^{2}}{C_{w}^{2}}} e^{-\frac{V_{z}^{2}}{C_{w}^{2}}}, \\
B_{y}\left(V^{\prime}, V\right)=\frac{1}{C_{w} \sqrt{\pi}} \delta\left(V_{x}+V_{x}^{\prime}\right) \delta\left(V_{y}-V_{y}^{\prime}\right) e^{-\frac{V_{z}^{2}}{C_{w}^{2}}} \\
\mathrm{~B}_{x}\left(V^{\prime}, V\right)=\frac{2}{C_{w}^{2}} V_{x} \delta\left(V_{y}-V_{y}^{\prime}\right) \delta\left(V_{z}-V_{z}^{\prime}\right) e^{-\frac{V_{x}^{2}}{C_{w}^{2}}}
\end{gathered}
$$

and

$$
\begin{array}{r}
\mu_{x z}=\alpha_{x} \alpha_{z}\left(1-\alpha_{y}\right), \quad \mu_{x y}=\alpha_{x} \alpha_{y}\left(1-\alpha_{z}\right), \quad \mu_{y z}=\alpha_{y} \alpha_{z}\left(1-\alpha_{x}\right) \\
\mu_{x}=\alpha_{x}\left(1-\alpha_{y}\right)\left(1-\alpha_{z}\right), \quad \mu_{x y z}=\alpha_{x} \alpha_{y} \alpha_{z}, \quad \mu_{0}=\left(1-\alpha_{x}\right)\left(1-\alpha_{y}\right)\left(1-\alpha_{z}\right) \\
\mu_{y}=\alpha_{y}\left(1-\alpha_{x}\right)\left(1-\alpha_{z}\right), \quad \mu_{z}=\alpha_{z}\left(1-\alpha_{x}\right)\left(1-\alpha_{y}\right) .
\end{array}
$$


A complete development of expression (15) yields the scattering kernel written as a sum of 32 elementary polyatomic scattering kernels $B_{\kappa} \tilde{P}_{i n}$ as follows:

$$
B=\sum_{\kappa, i n} \mu_{\kappa} \mu_{i n} B_{\kappa} \tilde{P}_{i n}
$$

where $\tilde{P}_{i n}$ refers to the four partial operators $\tilde{P}_{0}, \tilde{P}_{v}, \tilde{P}_{r}, \tilde{P}_{r v}$ defined in the relationship (A1) and $\mu_{i n}$ to their respective coefficients in the formula (A2). In this developed form it is clear that this scattering kernel describes various types of accommodation processes at the wall. Each of the partial scattering kernels $B_{\kappa} \tilde{P}_{i n}$ corresponds to a particular type of accommodation. There are exactly 32 types.

[1] R. G. Lord, Physic of Fluids 7, 1159 (1995).

[2] R. G. Lord, Physics of Fluid A 3, 706 (1991).

[3] I. Kuscer, in International Symposium on Rarefied Gas Dynamics, Gottingen (1974), vol. E1, pp. 1-21.

[4] C. Cercignani, R. Illner, and M. Pulvirenti, The Mathematical Theory of Dilute Gases (Springer-Verlag, 1994).

[5] S. K. Dadzie and J. G. Meolans, Journal of Mathematical Physics 45, 1804 (2004).

[6] C. Cercignani, Journal of Statistical Physics 1, 297 (1969).

[7] C. Cercignani, Mathematical Methods in Kinetic Theory (plenum press new york, 1990), chap. 4 , p. 98 .

[8] S. F. Shen, Entropie 18, 138 (1967).

[9] S. Nocilla, Meccanica 2, 34 (1967).

[10] C. Cercignani and M. Lampis, transport theory and statistical physics 1, 101 (1971).

[11] D.Bruno, M. Cacciatore, S. Longo, and M. Rutigliano, chemical physics letters 320, 245 $(2000)$. 